\title{
Orthogonal Symmetries and Reflections in Banach Spaces
}

\author{
Ali Jaballah and Fathi B. Saidi \\ University of Sharjah, Sharjah, UAE \\ Correspondence should be addressed to Ali Jaballah; ajaballah@sharjah.ac.ae \\ Received 5 March 2017; Accepted 12 April 2017; Published 14 June 2017 \\ Academic Editor: Ji Gao \\ Copyright (c) 2017 Ali Jaballah and Fathi B. Saidi. This is an open access article distributed under the Creative Commons Attribution \\ License, which permits unrestricted use, distribution, and reproduction in any medium, provided the original work is properly cited. \\ Let $X$ be a Banach space. We introduce a concept of orthogonal symmetry and reflection in $X$. We then establish its relation with \\ the concept of best approximation and investigate its implication on the shape of the unit ball of the Banach space $X$ by considering \\ sections over subspaces. The results are then applied to the space $C(I)$ of continuous functions on a compact set $I$. We obtain \\ some nontrivial symmetries of the unit ball of $C(I)$. We also show that, under natural symmetry conditions, every odd function is \\ orthogonal to every even function in $X$. We conclude with some suggestions for further investigations.
}

\section{Introduction}

When we try to imagine or picture a reflection of a point $x$ in a Banach space $X$ with respect to, say, a line $L$ passing through the origin, we tend to put ourselves in the context of a Euclidean space and think of a "mirror reflection" of the point $x$, that is, of a point $x^{r}$ that satisfies $\left\|x^{r}\right\|=\|x\| ; x$ and $x^{r}$ are equidistant from a point $m \in L\left(m=\left(x+x^{r}\right) / 2\right)$, such that, in addition, if we move $x$ and $x^{r}$ "away from $L$ " an equal distance, the two new points $x_{1}$ and $x_{1}^{r}$ still satisfy the same conditions; namely, $\left\|x_{1}^{r}\right\|=\left\|x_{1}\right\|$ and $x_{1}$ and $x_{1}^{r}$ are equidistant from $m$; see Definition 6 . It would be indeed nice and convenient if such a symmetry always existed, given its implications on the geometry of the unit ball of $X$. This would be a valuable asset that could help in establishing results in $X$ that otherwise may prove to be difficult. Moreover, there are many results in the literature that rely either directly or indirectly on the geometry of the unit ball of a Banach space. It may be interesting to revisit these and investigate the presence of such symmetries and their consequences. For some recent results in this direction, we refer the readers to $[1,2]$. We also note that the investigation of many concepts within Banach spaces is highly active. For some recent results along these lines we refer the readers for examples to $[3,4]$.

However, we all know that, in general, these "mirror symmetries" do not hold when we work inside a Banach space. So naturally, one should wonder about the types of spaces that do possess such symmetries. Our aim in this paper is to give necessary and sufficient conditions for this to be true and to investigate the consequences of these symmetries on the geometry of the unit ball of the Banach space; see Section 3. Before doing so, we investigate in Section 2 a weaker version of symmetry, which we term as "weak symmetry"; see Definition 1. We will establish a link between weak symmetry and the concept of best approximation (see Definition 2) and establish some of its properties and characteristics. In Section 4, we apply our results to the space $C(I)$ of continuous functions on a compact subset $I$ of $R$. We are able to obtain some nontrivial geometric regularity for the unit ball of $C(I)$. We also show that every odd function is orthogonal to every even function (Theorem 23). We conclude at the end of the paper by suggesting some directions for further investigations.

\section{Weak Symmetry in Banach Spaces}

Throughout this section, $X$ is a Banach space and $H$ is a closed subspace of $X$.

Definition 1. Let $H$ be a closed subspace of a Banach space $X$. An element $x^{r} \in X$ is called a weak-reflection point with respect to $H$ of the element $x \in X$ if

$$
\begin{gathered}
\frac{x+x^{r}}{2} \in H, \\
\left\|\frac{x-x^{r}}{2}\right\|=d(x, H) .
\end{gathered}
$$


If every element $x$ in a nonempty subset $A$ of $X$ admits a weak-reflection point $x^{r} \in A$ with respect to $H$, then we say that $A$ is weakly symmetric with respect to $H$.

We also need the following definition.

Definition 2. Let $H$ be a closed subspace of a Banach space $X$. An element $h_{o} \in H$ is called a best approximation from $H$ of an element $x \in X$ if it satisfies

$$
\left\|x-h_{o}\right\|=d(x, H):=\inf \{\|x-h\|: h \in H\} .
$$

If every element in $X$ admits a best approximation from $H$ then we say that $H$ is proximinal in $X$. The set of all best approximations of an element $x$ in $X$ is denoted by $P_{H}(x)$. The set-valued map $P_{H}: X \rightarrow 2^{H}$ is called the metric projection of $X$ onto $H$. If $P_{H}(x)$ is a singleton for every $x \in X$, then $H$ is said to be Chebyshev in $X$. If $P_{H}(x)$ is a singleton for some $x \in X$, then we denote the best approximation of $x$ also by $P_{H}(x)$.

It is clear that, in general, an element $x \in X$ may have more than one weak-reflection point with respect to $H$. In fact, we have the following characterization of uniqueness.

Lemma 3. Let $H$ be a closed subspace of a Banach space $X$. Then we have the following.

(i) An element $x \in X$ admits a weak-reflection point with respect to $H$ if and only if $P_{H}(x) \neq \emptyset$, in which case the set of weak-reflection points of $x$ is given by

$$
\begin{aligned}
& \left\{x^{r}: x^{r} \text { is a weak-refection point of } x\right\} \\
& \quad=2 P_{H}(x)-x .
\end{aligned}
$$

(ii) An element $x \in X$ admits a unique weak-reflection point with respect to $H$ if and only if $P_{H}(x)$ is a singleton.

(iii) Every element in $X$ admits a unique weak-reflection point with respect to $H$ if and only if $H$ is Chebyshev in $X$.

Proof. It follows directly from Definitions 1 and 2 that if an element $x \in X$ admits a weak-reflection point $x^{r}$ with respect to $H$ then

$$
h_{x}:=\frac{x+x^{r}}{2} \in P_{H}(x) ;
$$

hence $P_{H}(x) \neq \emptyset$. Conversely, suppose that $P_{H}(x) \neq \emptyset$ for some $x \in X$. Let $h_{x} \in P_{H}(x)$ and let

$$
x^{r}:=2 h_{x}-x .
$$

Then one easily checks that $x^{r}$ is a weak-reflection point of $x$. This completes the proof of Part (i). Parts (ii) and (iii) follow directly from Part (i). This ends the proof.

To explain the use of the term "weak" symmetry in Definition 1, we observe the following.

Lemma 4. Let $H$ be a closed subspace of a Banach space $X$. The following statements are equivalent. $H$. (ii) The subspace $H$ is proximinal in $X$.

(iii) For every $x \in X$, there exists an element $x^{r} \in X$ and a linear functional $f_{x} \in X^{*}$, where $X^{*}$ is the continuous dual space of $X$, such that

$$
\begin{gathered}
\|f\|=1, \\
H \subset \operatorname{ker} f, \\
\frac{x+x^{r}}{2} \in H, \\
f\left(x-x^{r}\right)=\left\|x-x^{r}\right\|,
\end{gathered}
$$

where ker $f$ denotes the kernel of the linear functional $f$.

Proof. (i) $\Leftrightarrow$ (ii): this follows directly from Lemma 3 .

(ii) $\Leftrightarrow$ (iii): this follows from Lemma 3 and Theorem 1.1 in [5].

It follows from the previous lemma that one can find a Banach space $X$ and a closed subspace $H$ of $X$ such that $X$ is not weakly symmetric with respect to $H$. In fact, it is well known that every nonreflexive Banach space $X$ admits a nonproximinal hyperplane $H$; see Corollary 2.4 in [5]; hence $X$ is not weakly symmetric with respect to $H$. An example of a nonreflexive Banach space is the Banach space BV $[0,1]$ of functions of bounded variation on the closed interval $[0,1]$ [6]. On the other hand, every reflexive subspace $H$ of a Banach space $X$ is proximinal; see Corollary 2.5 in [5]; hence a Banach space is always weakly symmetric with respect to its reflexive subspaces. In particular, every Banach space is weakly symmetric with respect to its finite dimensional subspaces. The following follows from the previous observations.

Remark 5. A Banach space $X$ is weakly symmetric with respect to all of its closed subspaces if and only if it is reflexive.

\section{Orthogonal Symmetry in Banach Spaces}

The notions of weak-reflection and weak symmetry that were discussed above are quite different from the "usual" notions of reflection and symmetry. As mentioned above, when we think about a reflection point $x^{r}$ of a point $x$ with respect to a subspace $H$ of a Banach space $X$, we tend to visualize (and would like it to be true) points $x^{r}$ and $x$ that are "mirror images" of each other with respect to $H$, that is, that are equidistant from the origin and from $H$, such that if we move them "away from $H$ " an equal distance, the two new points $x_{1}$ and $x_{1}^{r}$ still satisfy the same conditions. With this in mind, we introduce the following definition of symmetry.

Definition 6. Let $H$ be a closed subspace of a Banach space $X$. An element $x^{r} \in X$ is called a reflection point with respect to $H$ of the element $x \in X$ if

$$
\begin{aligned}
h_{x} & :=\frac{x+x^{r}}{2} \in H, \\
\left\|h_{x}+t\left(x-h_{x}\right)\right\| & =\left\|h_{x}+t\left(x^{r}-h_{x}\right)\right\|, \quad \forall t \in R .
\end{aligned}
$$


If every element $x$ in a nonempty subset $A$ of $X$ admits a reflection point $x^{r} \in A$ with respect to $H$, then we say that $A$ is symmetric with respect to $H$.

Setting $t=1$ in the second condition in Definition 6, we obtain immediately the following.

Remark 7. If $x^{r}$ is a reflection point with respect to $H$ of an element $x \in X$, then

$$
\left\|x^{r}\right\|=\|x\| .
$$

We recall the following notion of orthogonality, which was first introduced by Roberts [7].

Definition 8. Two elements $x$ and $y$ of a Banach space $X$ are said to be orthogonal (we write $x \perp y$ ) if, for every $\lambda \in R$,

$$
\|x+\lambda y\|=\|x-\lambda y\| \text {. }
$$

Two nonempty subsets $F$ and $G$ of $X$ are said to be orthogonal, and we write $F \perp G$, if every element of $F$ is orthogonal to every element of $G$. Given $x \in X$, we write $x \perp G$ if $x \perp g$ for every $g \in G$.

If $H$ is a closed subspace of a Banach space $X$ and $x \in X$, then we denote the set of orthogonal projections of $x$ onto $H$ by $P_{H}^{\perp}(x)$ :

$$
P_{H}^{\perp}(x):=\{h \in H:(x-h) \perp H\} .
$$

If $P_{H}^{\perp}(x)=\left\{h_{o}\right\}$ is a singleton, then we denote $h_{o}$ also by $P_{H}^{\perp}(x)$.

Note that this definition of orthogonality is symmetric in the sense that

$$
x \perp y \quad \text { iff } y \perp x .
$$

For a generalization of this notion of orthogonality to the case of orthogonal sequences in complex Banach spaces, we refer the reader to [8].

The following observation gives an alternative way of thinking about the notion of symmetry introduced in Definition 6 in terms of orthogonality.

Remark 9. Let $H$ be a closed subspace of a Banach space $X$. An element $x^{r} \in X$ is a reflection point with respect to $H$ of the element $x \in X$ if and only if $h_{x}:=\left(x+x^{r}\right) / 2$ is an orthogonal projection of the point $x$ onto $H$, that is, if and only if

$$
\begin{gathered}
h_{x}:=\frac{x+x^{r}}{2} \in H, \\
\left(x-h_{x}\right) \perp H .
\end{gathered}
$$

To address the question of uniqueness of reflection points (equivalently of orthogonal projections), we recall the following definition.

Definition 10. Let $A$ be a nonempty subset of a Banach space $X$. An element $c \in X$ is called a center of $A$ if $A$ is symmetric with respect to $c$, that is, if

$$
c-(a-c)=2 c-a \in A, \text { for every } a \in A .
$$

Note that $A$ is symmetric with respect to $c$ if and only if $A-c$ is symmetric with respect to the origin.

First we take a look at the uniqueness of centers. We have the following.

Lemma 11. If $A$ is a bounded nonempty subset of a Banach space $X$, then $A$ admits at most one center.

Proof. Suppose $A$ admits two distinct centers $c_{1}$ and $c_{2}$. Let $R_{i}$ denote the reflection with respect to $c_{i}, i=1,2$. For each $a \in A$, we have $R_{i}(a)-c_{i}=c_{i}-a$; hence

$$
R_{i}(a)=2 c_{i}-a, \quad i=1,2 .
$$

It follows that

$$
\begin{aligned}
\left(R_{1} R_{2}\right)(a) & =R_{1}\left(2 c_{2}-a\right)=2 c_{1}-\left(2 c_{2}-a\right) \\
& =a-2\left(c_{2}-c_{1}\right) .
\end{aligned}
$$

By induction we obtain that

$$
\left(R_{1} R_{2}\right)^{n}(a)=a-2 n\left(c_{2}-c_{1}\right), \quad a \in A, n \geq 1 .
$$

Similarly we show that

$$
\left(R_{2} R_{1}\right)^{n}(a)=a+2 n\left(c_{2}-c_{1}\right), \quad a \in A, n \geq 1 .
$$

Now let $a \in A$ be fixed and consider the two sequences of points in $A$ defined by

$$
\begin{aligned}
x_{n}:=\left(R_{1} R_{2}\right)^{n}(a), & \\
y_{n}:=\left(R_{2} R_{1}\right)^{n}(a), & \\
& n=1,2, \ldots .
\end{aligned}
$$

It follows from (16) and (17) that

$$
\left\|y_{n}-x_{n}\right\|=4 n\left\|c_{2}-c_{1}\right\| \longrightarrow \infty, \quad \text { as } n \longrightarrow \infty .
$$

This implies that $A$ is unbounded, which contradicts the assumption in the lemma. This ends the proof. lemma.

The following follows from the proof of the previous

Corollary 12. Let $A$ be a nonempty subset of a Banach space $X$. If $A$ admits two distinct centers, then $A$ is unbounded.

We note that there are various notions of centers in the literature and that they all play an important role in approximation theory and in geometric functional analysis in general.

We have seen above in Section 2 that, in general, weakreflection points are not unique and that a weak-reflection point of an element $x \in X$ with respect to a closed subspace $H$ is unique if and only if $P_{H}(x)$ is a singleton. The situation is quite different in the case of symmetry. The following theorem shows, in particular, that the concept of symmetry is stronger than the concept of weak symmetry and that reflection points and orthogonal projections, when they exist, are unique. 
Theorem 13. Let $H$ be a closed subspace of a Banach space X. Then we have the following.

(i) If $X$ is symmetric with respect to $H$, then $X$ is weakly symmetric with respect to $H$.

(ii) Every element $x \in X$ admits at most one orthogonal projection $P_{H}^{\perp}(x)$ onto $H$. Moreover, if an element $x \in X$ admits an orthogonal projection $P_{H}^{\perp}(x)$ onto $H$ then the set of best approximations $P_{H}(x)$ admits a unique center $c_{x}, x$ admits a unique reflection point $x^{r}$ with respect to $H$, and the reflection point $x^{r}$ of $x$ is given by

$$
x^{r}=2 c_{x}-x=2 P_{H}^{\perp}(x)-x .
$$

(iii) Every element $x$ in $X$ admits at most one reflection point $x^{r}$ with respect to $H$. Moreover, if an element $x \in$ $X$ admits a reflection point $x^{r}$ with respect to $H$, then set of best approximations $P_{H}(x)$ admits a unique center $c_{x}, x$ admits a unique orthogonal projection $P_{H}^{\perp}(x)$ onto $H$, and the orthogonal projection of $x$ onto $H$ is given by

$$
P_{H}^{\perp}(x)=c_{x}=\frac{x+x^{r}}{2} .
$$

Proof. (i) Suppose that $X$ is symmetric with respect to $H$. Let $x \in X$ be given and let $x^{r}$ be a reflection point of $x$. We will show that $x^{r}$ is a weak-reflection point of $x$. Indeed we have, by Remark 9 ,

$$
\begin{gathered}
h_{x}:=\frac{x+x^{r}}{2} \in H, \\
\left(x-h_{x}\right) \perp H .
\end{gathered}
$$

Hence it suffices to show that $\left\|x-h_{x}\right\|=d(x, H)$. For every $h \in H$ we have, since $\left(x-h_{x}\right) \perp H$,

$$
\begin{aligned}
\|x-h\| & =\left\|z_{1}:=x-h_{x}-\left(h-h_{x}\right)\right\| \\
& =\left\|z_{2}:=x-h_{x}+\left(h-h_{x}\right)\right\| .
\end{aligned}
$$

It follows, since $\|x\|$ is a convex function of $x$, that

$$
\left\|x-h_{x}\right\|=\left\|\frac{z_{1}+z_{2}}{2}\right\| \leq \frac{\left\|z_{1}\right\|+\left\|z_{2}\right\|}{2}=\|x-h\|,
$$

for every $h \in H$.

Hence $\left\|x-h_{x}\right\|=d(x, H)$ and the proof of Part (i) is complete.

(ii) Let $x \in X$ be fixed and suppose that $x$ admits an orthogonal projection $h_{x}$ onto $H$; hence

$$
\left(x-h_{x}\right) \perp H .
$$

Then, by Remark 9, $x$ admits a reflection point and hence, by Part (i) and Lemma 3, $P_{H}(x) \neq \varnothing$. We now show that $h_{x}$ is a center of $P_{H}(x)$. We need to show that $\left(2 h_{x}-h\right) \in P_{H}(x)$, for every $h \in P_{H}(x)$. Let $h \in P_{H}(x)$ be given. It follows, since $\left(h_{x}-h\right) \in H$ and since $\left(x-h_{x}\right) \perp H$, that

$$
\begin{aligned}
\left\|x-\left(2 h_{x}-h\right)\right\| & =\left\|\left(x-h_{x}\right)-\left(h_{x}-h\right)\right\| \\
& =\left\|\left(x-h_{x}\right)+\left(h_{x}-h\right)\right\|=\|x-h\| \\
& =d(x, H) .
\end{aligned}
$$

Hence $\left(2 h_{x}-h\right) \in P_{H}(x)$ for every $h \in P_{H}(x)$. This implies that $h_{x}$ is indeed a center of $P_{H}(x)$. It follows, by Lemma 11 and since $P_{H}(x)$ is bounded, that $h_{x}$ is the unique center $c_{x}$ of $P_{H}(x)$. Hence the orthogonal projection of $x$ onto $H$ is unique and is given by

$$
P_{H}^{\perp}(x)=c_{x} .
$$

It follows from Remark 9 that $x^{r}:=2 P_{H}^{\perp}(x)-x$ is a reflection point of $x$ and that it is unique since, again by Remark 9, $\left(x^{r}+x\right) / 2$ must be an orthogonal projection of $x$ onto $H$. This completes the proof of Part (ii).

(iii) This follows directly from Part (ii) and Remark 9. This completes the proof of the theorem.

The following follows immediately from Theorem 13.

Corollary 14. A Banach space $X$ is symmetric with respect to a closed subspace $H$ if and only if every element $x \in X$ admits an orthogonal projection onto $H$.

Remark 15. For every element $x \in X, P_{H}(x)$ is always a convex subset of the subspace $H$ of $X$; hence the center $c_{x}$ and the orthogonal projection $P_{H}^{\perp}(x)$ always belong to $P_{H}(x)$ whenever they exist.

It is important to note that $H$ does not have to be Chebyshev in $X$ in order for $X$ to be symmetric with respect to $H$. Indeed we have the following.

Example 16. Consider the Banach space

$$
(X,\|\cdot\|):=\left(R^{2},\|\cdot\|_{\infty}\right)
$$

where $\|(a, b)\|_{\infty}:=\max \{|a|,|b|\}$, and let

$$
H:=\{(0, b): b \in R\} .
$$

Then we have, for every $(a, b) \in X$,

$$
P_{H}(a, b)=\{(0, b+\lambda a): \lambda \in[-1,1]\} ;
$$

hence $H$ is proximinal but not Chebyshev in $X$. Also, one can easily verify that $X$ is symmetric with respect to $H$ and that, for every $x:=(a, b) \in X$, the unique center $c_{x}$ of $P_{H}(x)$ and the unique reflection point $x^{r}$ of $x$ are given by

$$
\begin{aligned}
& c_{x}=(0, b), \\
& x^{r}=(-a, b) .
\end{aligned}
$$

We also point out that the existence of a center of $P_{H}(x)$ does not guarantee the existence of a reflection point (or of an orthogonal projection) of $x$. Indeed we have the following.

Example 17. Let $X$ and $H$ be as in Example 16 but, instead of $\|\cdot\|_{\infty}$, consider the norm

$$
\|(a, b)\|:= \begin{cases}\sqrt{a^{2}+b^{2}} & \text { if } a b \geq 0 \\ |a|+|b| & \text { if } a b<0 .\end{cases}
$$


Note that $\|\cdot\|$ is the Minkowski functional associated with the closed convex and symmetric subset $K$ of $X$ given by

$$
K:=\{(a, b) \in X:\|(a, b)\| \leq 1\} ;
$$

hence indeed it is a norm on $X$. To verify this, all we need to check is the triangle inequality as the other properties are trivial: First, recall that we always have, for all $s, t \in R$,

$$
|| s|-| t|| \leq \sqrt{s^{2}+t^{2}} \leq|s|+|t|
$$

hence, for all $s, t \in R$,

$$
\left\|s|-| t|| \leq \sqrt{s^{2}+t^{2}} \leq\right\|(s, t) \| \leq|s|+|t| .
$$

Also, for all $s_{1}, s_{2}, t_{1}$, and $t_{2}$ in $R$, we have

$$
\sqrt{\left(s_{1}+s_{2}\right)^{2}+\left(t_{1}+t_{2}\right)^{2}} \leq \sqrt{s_{1}^{2}+t_{1}^{2}}+\sqrt{s_{2}^{2}+t_{2}^{2}}
$$

Now, let $x=(a, b)$ and $y=(c, d)$ be two arbitrary elements in $X$.

If $(a+c)(b+d) \geq 0$, then we have, by (35) and (36),

$$
\begin{aligned}
\|x+y\| & =\sqrt{(a+c)^{2}+(b+d)^{2}} \\
& \leq \sqrt{a^{2}+b^{2}}+\sqrt{c^{2}+d^{2}} \leq\|x\|+\|y\| .
\end{aligned}
$$

If $(a+c)(b+d)<0$, then one of the factors is positive and one is negative, say

$$
\begin{aligned}
& a+c>0, \\
& b+d<0 .
\end{aligned}
$$

It follows, by (35), that

$$
\begin{aligned}
\|x+y\| & =|a+c|+|b+d|=a+c-b-d \\
& =(a-b)+(c-d) \leq\|x\|+\|y\|,
\end{aligned}
$$

where the last inequality follows from the fact that

$$
\begin{aligned}
& (a-b) \\
& \leq \begin{cases}\max \{|a|,|b|\} \leq \sqrt{a^{2}+b^{2}} \leq\|(a, b)\| & \text { if } a b \geq 0 \\
|a|+|b|=\|(a, b)\| & \text { if } a b<0,\end{cases}
\end{aligned}
$$

and we can also easily obtain a similar inequality for $(c-d)$. Going back to our example, it is clear that, for every $(a, b) \in$ $X$,

$$
P_{H}(a, b)=\{(0, b)\} .
$$

Hence $H$ is Chebyshev in $X$ and, for each $x:=(a, b) \in X$, $P_{H}(x)$ admits a unique center, namely, $c_{x}=(0, b)$. It follows from Theorem 13 that if an element $x:=(a, b)$ in $X$ admitted a reflection point $x^{r}$ with respect to $H$, then we would have $x^{r}=2 c_{x}-x=(-a, b)$ and, by Remark 9 , we would also have $\left(x-c_{x}\right) \perp H$ or, in other words,

$$
(a, 0) \perp H \text {. }
$$

But clearly this is not possible if $a \neq 0$. Indeed, in this case $h:=(0, a) \in H$ and

$$
\|(a, 0)+h\|=\|(a, a)\|=|a| \sqrt{2},
$$

while

$$
\|(a, 0)-h\|=\|(a,-a)\|=2|a| \neq|a| \sqrt{2} .
$$

Hence (42) does not hold and consequently $(a, b)$ does not have a reflection point if $a \neq 0$.

We now highlight the effect of symmetry on the geometry of the unit ball $B_{X}$ of the Banach space $X$ by looking at its 1-dimensional sections over the subspace $H$, that is, by looking at sections of $B_{X}$ by subspaces of $X$ in which $H$ is of codimension 1 .

Theorem 18. If a Banach space $X$ is symmetric with respect to a proper closed subspace $H$, then its unit ball $B_{X}$ is symmetric with respect to $H$. Moreover, given any point $y \in X \backslash H$, the subspace $H$ divides the unit ball $B_{F}$ of $F:=\operatorname{span}\{y, H\}$ into two halves which are identical modulo reflection with respect to $H$. The two halves of $B_{F}$ are given by

$$
\begin{aligned}
B_{F}^{ \pm} & :=B_{F} \cap\left\{h \pm|\alpha|\left(y-c_{y}\right): h \in H, \alpha \in R\right\} \\
& =B_{F} \cap\{h \pm|\alpha| y: h \in H, \alpha \in R\},
\end{aligned}
$$

where $c_{y}$ is the unique center of $P_{H}(y)$.

Proof. Since $X$ is symmetric with respect to $H$, it follows immediately from Remark 7 that $B_{X}$ is symmetric with respect to $H$. Now let $x \in B_{X}$ be given. It follows from Remark 9 and Theorem 13 that

$$
\begin{gathered}
c_{x}=\frac{x+x^{r}}{2} \in H, \\
\left(x-c_{\mathrm{x}}\right) \perp H,
\end{gathered}
$$

where $x^{r}$ is the reflection point of $x$. If $x \in B_{F} \subset B_{X}$, then we have

$$
x^{r}=2 c_{x}-x \in \operatorname{span}\{x, H\}:=F .
$$

Hence $x^{r} \in B_{X} \cap F=B_{F}$. Therefore $B_{F}$ is symmetric with respect to $H$. Since $H$ is a hyperplane in $F, H$ divides $B_{F}$ into two halves, $B_{F}^{+}$and $B_{F}^{-}$. Clearly, if $x:=h+|\alpha|\left(y-c_{y}\right) \in B_{F}^{+}$for some $h \in H$ and $\alpha \in R$, then the reflection point of $x$ is given by

$$
x^{r}:=h-|\alpha|\left(y-c_{y}\right) \in B_{F}^{-} .
$$

This follows from Remark 9 and from the fact that $\left(y-c_{y}\right) \perp$ $H$. Hence $B_{F}^{-}$is the reflection of $B_{F}^{+}$. Similarly we show that $B_{F}^{+}$ is the reflection of $B_{F}^{-}$. This ends the proof.

One should note that the orthogonal symmetry of the unit ball given by Theorem 18 is not always true in Banach spaces. It follows from Definition 6 and Remark 9 that it holds if and only if the unit ball $B_{X}$ of $X$ is symmetric with respect 
to $H$ in the sense of Definition 6. In particular, it is possible for a Banach space $X$ to be weakly symmetric with respect to a closed subspace $H$, while the unit ball $B_{X}$ is neither symmetric nor weakly symmetric with respect to $H$. Indeed we have the following.

Example 19. Consider again the example given in Example 17 above. Then $X$ is weakly symmetric with respect to $H$, since $H$ is Chebyshev in $X$. It is easy to verify that

$$
x:=\left(\frac{\sqrt{2}}{2}, \frac{\sqrt{2}}{2}\right) \in B_{X}
$$

but the weak reflection point

$$
x^{r}=\left(-\frac{\sqrt{2}}{2}, \frac{\sqrt{2}}{2}\right) \notin B_{X} .
$$

Hence $B_{X}$ is neither weakly symmetric nor symmetric with respect to $H$.

We now consider the case where $X$ is the direct sum of two mutually orthogonal subspaces.

Theorem 20. Suppose that a Banach space $X$ is the direct sum of two mutually orthogonal closed subspaces $H$ and $G$ :

$$
\begin{gathered}
H \perp G, \\
H \oplus G=X .
\end{gathered}
$$

Then $X$ is symmetric with respect to both $H$ and $G$ and we have, for each $x \in X$,

$$
\begin{aligned}
x & =P_{H}^{\perp}(x)+P_{G}^{\perp}(x), \\
x_{H}^{r} & =P_{H}^{\perp}(x)-P_{G}^{\perp}(x), \\
x_{G}^{r} & =P_{G}^{\perp}(x)-P_{H}^{\perp}(x),
\end{aligned}
$$

where $x_{H}^{r}$ and $x_{G}^{r}$ are the reflections of $x$ with respect to $H$ and $G$, respectively.

Proof. Let $x \in X$. Then $x=h+g$, for some $h \in H$ and $g \in G$. Now let $x_{H}^{r}:=h-g$. Then we have, since $G \perp H$ and $x-h=g \in G$,

$$
\begin{gathered}
\frac{x+x_{H}^{r}}{2}=h \in H, \\
(x-h) \perp H .
\end{gathered}
$$

Hence, by Remark 9, $x_{H}^{r}$ is a reflection point of $x$ with respect to $H$ and, by Theorem 13 ,

$$
P_{H}^{\perp}(x)=\frac{x+x_{H}^{r}}{2}=h .
$$

Since $x$ was arbitrary in $X, X$ is symmetric with respect to $H$. Similarly we show that $x_{G}^{r}:=g-h$ is a reflection point of $x$ with respect to $G$, that $X$ is symmetric with respect to $G$, and that

$$
P_{G}^{\perp}(x)=\frac{x+x_{G}^{r}}{2}=g .
$$

Hence

$$
P_{H}^{\perp}(x)+P_{G}^{\perp}(x)=\frac{x+x_{H}^{r}}{2}+\frac{x+x_{G}^{r}}{2}=x,
$$

since $x_{H}^{r}+x_{G}^{r}=0$. This ends the proof.

The reader may be wondering about the situation when a Banach space $X$ is symmetric with respect to all of its closed subspaces. For one thing, it follows from Remark 5 and Theorem 13 that $X$ must be reflexive. Also it is clear that this condition cannot be sufficient, as symmetry and weak symmetry are not equivalent. It turns out that the situation is possible only if $X$ is isometric to a Hilbert space. We have the following stronger result.

Theorem 21. Let $X$ be a Banach space satisfying $\operatorname{dim} X \geq 2$. Then $X$ is symmetric with respect to all of its 1-dimensional closed subspaces if and only if it is isometric to a Hilbert space.

Proof. Suppose $X$ is symmetric with respect to all of its 1-dimensional closed subspaces. Let $F$ be an arbitrary 2dimensional subspace of $X$, let $x$ be a nonzero element in $F$, and let

$$
H:=\operatorname{span}\{x\}:=\{r x: r \in R\} .
$$

We first show that $x$ admits a nonzero orthogonal element in $F$. Let $y \in F$ be linearly independent of $x$. Since $H$ is 1-dimensional, $X$ is symmetric with respect to $H$ by assumption. Therefore there exists $h_{y} \in H$ such that $\left(y-h_{y}\right) \perp$ $H$. It follows, since $x \in H$ and $y \notin H$, that

$$
\begin{gathered}
\left(y-h_{y}\right) \neq 0, \\
\left(y-h_{y}\right) \perp x, \\
\left(y-h_{y}\right) \in \operatorname{span}\{x, y\}=F .
\end{gathered}
$$

Therefore we have proved that in every 2-dimensional subspace of $X$ every nonzero element admits a nonzero orthogonal element. It follows by a theorem of James [9] that $X$ is isometric to a Hilbert space. This ends the proof.

\section{A Final Remark}

Remark 22. Since, by Remark 7, the concept of symmetry given by Definition 6 is norm-preserving, it follows immediately that in Theorem 18 we may replace the unit balls by balls or spheres of radius $\rho$.

\section{Symmetries of the Space $C(I)$}

Let $I$ be a nonempty compact subset of the set of real numbers $R, I$ containing at least two elements, and let $C(I)$ be the 
Banach space of real-valued continuous functions defined on $I$, where the norm of an element $f \in C(I)$ is given by

$$
\|f\|:=\sup \{|f(x)|: x \in I\}:=\sup _{I}|f| .
$$

Given $f \in C(I)$ and $c \in R$, we let $f_{c}$ be the element of $C\left(I_{c}\right)$ defined by

$$
\begin{array}{r}
f_{c}(x):=f(x+c), \\
x \in I_{c}:=I-c .
\end{array}
$$

Note that $f_{c} \in C\left(I_{c}\right)$ whenever $f \in C(I)$. If $I$ is symmetric with respect to some $c \in R$, we denote by $E_{c}(I)$ and $O_{c}(I)$ the closed subspaces of $C(I)$ given by

$$
\begin{aligned}
& E_{c}(I):=\left\{f \in C(I): f_{c} \text { is an even function }\right\}, \\
& O_{c}(I):=\left\{f \in C(I): f_{c} \text { is an odd function }\right\} .
\end{aligned}
$$

When $c=0$, we let

$$
\begin{aligned}
& O(I):=O_{0}(\mathrm{I}), \\
& E(I):=E_{0}(I) .
\end{aligned}
$$

With these definitions in mind, we have the following.

Theorem 23. If I is symmetric with respect to some $c \in R$, then $E_{c}(I) \perp O_{c}(I)$ and $C(I)$ is the direct sum of $E_{c}(I)$ and $O_{c}(I)$ :

$$
C(I)=E_{c}(I) \oplus O_{c}(I) ;
$$

hence $C(I)$ is symmetric with respect to both $E_{c}(I)$ and $O_{c}(I)$. Moreover, for each $f:=f_{e} \oplus f_{o} \in E_{c}(I) \oplus O_{c}(I)=C(I)$, we have

$$
\begin{aligned}
f_{E_{c}(I)}^{r} & =f_{e}-f_{o}, \\
f_{O_{c}(I)}^{r} & =f_{o}-f_{e}, \\
\text { hence } f_{E_{c}(I)}^{r} & =-f_{O_{c}(I)}^{r},
\end{aligned}
$$

where $f_{E_{c}(I)}^{r}$ and $f_{\mathrm{O}_{c}(I)}^{r}$ are the reflections of $f$ with respect to $E_{c}(I)$ and $O_{c}(I)$, respectively.

Proof. First we prove that $E_{c}(I)$ is orthogonal to $O_{c}(I)$ : Let $h \in E_{c}(I)$ and $g \in O_{c}(I)$ be given. Since $h_{c}$ is an even function, $g_{c}$ is an odd function, and $-I_{c}=I_{c}$; we have

$$
\begin{aligned}
\|h+\operatorname{tg}\| & :=\sup _{x \in I}\|h(x)+\operatorname{tg}(x)\| \\
& =\sup _{x \in I_{c}}\left\|h_{c}(x)+\operatorname{tg}_{c}(x)\right\| \\
& =\sup _{x \in-I_{c}}\left\|h_{c}(-x)+\operatorname{tg}_{c}(-x)\right\| \\
& =\sup _{x \in I_{c}}\left\|h_{c}(x)-\operatorname{tg}_{c}(x)\right\| \\
& =\sup _{x \in I}\|h(x)-\operatorname{tg}(x)\|=\|h-\operatorname{tg}\|,
\end{aligned}
$$

for every $t \in R$. Hence

$$
E_{c}(I) \perp O_{c}(\mathrm{I}) .
$$

Now, let $f \in C(I)$ be given. Then

$$
\begin{aligned}
f(x) & =\frac{f(x)+f(2 c-x)}{2}+\frac{f(x)-f(2 c-x)}{2} \\
& :=f_{e}(x)+f_{o}(x), \quad \forall x \in I .
\end{aligned}
$$

Note that

$$
\begin{aligned}
& f_{e} \in E_{c}(I), \\
& f_{o} \in O_{c}(I) .
\end{aligned}
$$

It follows that $C(I)=E_{c}(I)+O_{c}(I)$ and, since $E_{c}(I) \cap O_{c}(I)=$ $\{0\}$, that

$$
C(I)=E_{c}(I) \oplus O_{c}(I) .
$$

The theorem now follows from Theorem 20. This completes the proof.

Note that Theorems 23 and 18 shed some light on the shape and geometry of the unit ball of the Banach space $C(I)$, which otherwise are not easy to visualize. In general the only guaranteed symmetry in a Banach space is symmetry with respect to the origin. But here, with the help of Theorems 23 and 18, one can actually visualize sections of the unit ball of $C(I)$. Indeed, if we consider, for example, a 2-dimensional section of the unit ball, say of $C(-1,1)$, by a 2-dimensional subspace $F:=\operatorname{span}\{f, g\}$, where $f(\neq 0) \in O(-1,1)$ and $g(\neq$ $0) \in E(-1,1)$, then Theorem 23 tells us that the two lines $L_{f}:=$ $\operatorname{span}\{f\}$ and $L_{g}:=\operatorname{span}\{g\}$ divide the 2-dimensional unit sphere of $F$ into four identical quarters modulo reflections with respect to $L_{f}$ and $L_{g}$. Note that this characteristic is not always true in Banach spaces. More generally, the following follows immediately from Theorems 23 and 18.

Corollary 24. Let I be symmetric with respect to some $c \in R$ and let $H$ represent one of the two subspaces $O_{c}(I)$ and $E_{c}(I)$. Then, for every $g \in C(I) \backslash H, H$ divides each of the unit sphere $S_{F}$ and the unit ball $B_{F}$ of the subspace $F:=\operatorname{span}\{g, H\}$ of $C(I)$ into two identical halves modulo reflection with respect to $H$. The two halves of $S_{F}$ and $B_{F}$ are given by, respectively,

$$
\begin{aligned}
& S_{F}^{ \pm}:=S_{F} \cap\{\alpha h \pm|\beta| g: h \in H, \alpha, \beta \in R\}, \\
& B_{F}^{ \pm}:=B_{F} \cap\{\alpha h \pm|\beta| g: h \in H, \alpha, \beta \in R\} .
\end{aligned}
$$

We note that there are symmetries in $C(I)$ other than those mentioned above. Indeed, let $|I|$ denote the cardinality of $I$ and, for each $f \in C(I)$, let $\operatorname{supp}(f)$ be defined by

$$
\operatorname{supp}(f):=\{t \in I: f(t) \neq 0\} \text {. }
$$

Then we have the following.

Proposition 25. Let $I$ be symmetric with respect to some $c \in$ $R,|I| \geq 4$, and let $\{H, K\}:=\left\{O_{c}(I), E_{c}(I)\right\}$. Choose any two elements $k_{1}$ and $k_{2}$ from the unit sphere $S_{K}$ of $K$ such that

$$
\operatorname{supp}\left(k_{1}\right) \cap \operatorname{supp}\left(k_{2}\right)=\emptyset .
$$


Now let $F=\operatorname{span}\left\{H, k_{1}, k_{2}\right\}, F_{1}:=\operatorname{span}\left\{H, k_{1}\right\}$, and $F_{2}=$ $\operatorname{span}\left\{H, k_{2}\right\}$. Then

$$
\begin{gathered}
F_{i} \perp k_{j}, \\
F=F_{i} \oplus \operatorname{span}\left\{k_{j}\right\}, \\
\{i, j\}=\{1,2\} .
\end{gathered}
$$

Moreover, the hyperplanes $F_{1}$ and $F_{2}$ of $F$ together divide each of the unit sphere $S_{F}$ and the unit ball $B_{F}$ of $F$ into four identical quarters modulo reflections with respect to $F_{1}$ and $F_{2}$. The four quarters of the unit sphere $S_{F}$ and the unit ball $B_{F}$ of $F$ are given by, respectively,

$$
\begin{aligned}
S_{F}^{ \pm \pm}:= & S_{F} \\
& \cap\left\{\alpha h \pm|\beta| k_{1} \pm|\gamma| k_{2}: h \in H, \alpha, \beta, \gamma \in R\right\}, \\
B_{F}^{ \pm \pm}= & B_{F} \\
& \cap\left\{\alpha h \pm|\beta| k_{1} \pm|\gamma| k_{2}: h \in H, \alpha, \beta, \gamma \in R\right\} .
\end{aligned}
$$

Proof. First note that, since $|I| \geq 4$, one can indeed choose two elements $k_{1}$ and $k_{2}$ from the unit sphere $S_{K}$ of $K$ such that

$$
\operatorname{supp}\left(k_{1}\right) \cap \operatorname{supp}\left(k_{2}\right)=\emptyset .
$$

Let $I_{j}:=\operatorname{supp}\left(k_{j}\right), j=1,2$, and let $I_{3}:=I \backslash\left(I_{1} \cup I_{2}\right)$. Then $I$ is the disjoint union of $I_{1}, I_{2}$, and $I_{3}$. Note that, since $\left\|k_{1}\right\|=$ $\left\|k_{2}\right\|=1, I_{1}$ and $I_{2}$ are nonempty. Since $I$ is symmetric with respect to $c$ and $k_{1}, k_{2} \in K$, it follows that

$$
I_{j} \text { is symmetric with respect to } c, j=1,2,3 \text {. }
$$

First, we show that $F_{i} \perp k_{j},\{i, j\}=\{1,2\}$. Let $\left(h+\alpha k_{i}\right) \in F_{i}$ be fixed. Then we have, since $I_{1}, I_{2}$, and $I_{3}$ have disjoint supports,

$$
\begin{aligned}
& \left\|h+\alpha k_{i}+\lambda k_{j}\right\|:=\sup _{I}\left|h+\alpha k_{i}+\lambda k_{j}\right| \\
& \quad=\max \left\{\sup _{I_{i}}\left|h+\alpha k_{i}\right|, \sup _{I_{j}}\left|h+\lambda k_{j}\right|, \sup _{I_{3}}|h|\right\} .
\end{aligned}
$$

Since $I_{j}$ is nonempty and symmetric with respect to $c$, so is the closure $\mathrm{cl}\left(I_{j}\right)$ of $I_{j}$. It follows from Theorem 23 that $O_{c}\left(\mathrm{cl}\left(I_{j}\right)\right) \perp E_{c}\left(\mathrm{cl}\left(I_{j}\right)\right)$ and hence the restrictions of $h$ and $k_{j}$ to $\mathrm{cl}\left(I_{j}\right)$ are orthogonal in $C\left(\mathrm{cl}\left(I_{j}\right)\right)$. This implies that

$$
\begin{aligned}
\sup _{I_{j}}\left|h+\lambda k_{j}\right| & =\sup _{\operatorname{cl}\left(I_{j}\right)}\left|h+\lambda k_{j}\right|=\sup _{\operatorname{cl}\left(I_{j}\right)}\left|h-\lambda k_{j}\right| \\
& =\sup _{I_{j}}\left|h-\lambda k_{j}\right| .
\end{aligned}
$$

It follows that

$$
\begin{aligned}
\| h & +\alpha k_{i}+\lambda k_{j} \| \\
& =\max \left\{\sup _{I_{i}}\left|h+\alpha k_{i}\right|, \sup _{I_{j}}\left|h-\lambda k_{j}\right|, \sup _{I_{3}}|h|\right\} \\
& =\sup _{I}\left|h+\alpha k_{i}-\lambda k_{j}\right|=\left\|h+\alpha k_{i}-\lambda k_{j}\right\| ;
\end{aligned}
$$

hence $\left(h+\alpha k_{i}\right) \perp k_{j}$ and consequently $F_{i} \perp k_{j}$, as required.
To prove that $F=F_{i} \oplus \operatorname{span}\left\{k_{j}\right\},\{i, j\}=\{1,2\}$, it suffices to show that $k_{j} \notin F_{i}$. But this follows directly from the fact that $k_{j} \neq 0$ and $k_{j} \perp F_{i}$.

Note that $k_{1}$ and $k_{2}$ are linearly independent. Therefore, since $E_{c}(I) \perp O_{c}(I)$,

$$
\begin{aligned}
& F_{1}:=\operatorname{span}\left\{k_{1}, H\right\} \perp \operatorname{span}\left\{k_{2}\right\}, \\
& F=F_{1} \oplus \operatorname{span}\left\{k_{2}\right\} .
\end{aligned}
$$

Also

$$
\begin{gathered}
F_{2}: \operatorname{span}\left\{k_{2}, H\right\} \perp \operatorname{span}\left\{k_{1}\right\}, \\
F=F_{2} \oplus \operatorname{span}\left\{k_{1}\right\} .
\end{gathered}
$$

It follows from Theorem 18 that the hyperplanes $F_{1}$ and $F_{2}$ of $F$ together divide the unit sphere and the unit ball of $F$ into four identical quarters modulo reflections with respect to $F_{1}$ and $F_{2}$. The four quarters of the unit sphere $B_{F}$ of $F$ are

$$
\begin{aligned}
B_{F}^{ \pm \pm}:= & B_{F} \\
& \cap\left\{\alpha h \pm|\beta| k_{1} \pm|\gamma| k_{2}: h \in H, \alpha, \beta, \gamma \in R\right\} .
\end{aligned}
$$

Similarly we obtain the four quarters of the unit sphere $S_{F}$ of F. tions:

We conclude with some suggestions for further investiga-

(1) With Theorem 18 in mind, one may look at $n$ dimensional or infinite-dimensional sections of the unit ball of the Banach space $X$ over the subspace $H$, $n \geq 2$. For this one may need to consider the extension of the notion of orthogonality introduced in [8].

(2) Instead of a general Banach space, we may consider specific Banach spaces $X$ and identify the subspaces $H$ such that $X$ is symmetric with respect to $H$. For instance, it is not difficult to show that if $X$ is the usual $l^{p}$ sequence-space, $1 \leq p \leq \infty$, then $X$ is orthogonally symmetric with respect to all subspaces $H$ spanned by the coordinate-vectors, $H:=\operatorname{span}\left\{e_{i}: i \in J\right\}$, where $J$ is a nonempty finite or infinite subset of positive integers and $e_{i}$ is the usual coordinate vector with 1 in the $i$ th-place and zeros elsewhere. Still, are there other subspaces with respect to which $X$ is symmetric?

(3) As mentioned above in Introduction, there are many results in the literature that rely either directly or indirectly on the geometry of the unit ball of a Banach space. It may be interesting to revisit these and investigate the presence of orthogonal symmetries and their consequences.

\section{Conflicts of Interest}

The authors declare that they have no conflicts of interest.

\section{References}

[1] M. Sababheh and R. Khali, "Remotal points and a KreinMilman type theorem," Journal of Nonlinear and Convex Analysis. An International Journal, vol. 12, no. 1, pp. 5-15, 2011. 
[2] R. Khalil, "Orthogonality in Banach spaces," Mathematics Journal of Toyama University, vol. 13, pp. 185-205, 1990.

[3] C. Asci, "Differentiation theory over infinite-dimensional banach spaces," Journal of Mathematics, vol. 2016, Article ID 2619087, 16 pages, 2016.

[4] S. Wulede, W. Bai, and W. Bao, "A generalization of uniformly extremely convex Banach spaces," Journal of Function Spaces, vol. 2016, Article ID 9161252, 6 pages, 2016.

[5] I. Singer, Best Approximation in Normed Linear Spaces by Elements of Linear Subspaces, Springer-Verlag, New York, NY, USA, 1970.

[6] F. Prus-Wis'niowski and W. H. Ruckle, "The Banach spaces $\Lambda \mathrm{BV}$ are non-reflexive," Journal of Mathematical Analysis and Applications, vol. 389, no. 2, pp. 1394-1396, 2012.

[7] B. D. Roberts, "On the geometry of abstract vector spaces," Tohoku Mathematical Journal, vol. 39, pp. 52-59, 1934.

[8] F. B. Saidi, "An extension of the notion of orthogonality to Banach spaces," Journal of Mathematical Analysis and Applications, vol. 267, no. 1, pp. 29-47, 2002.

[9] R. C. James, "Orthogonality in normed linear spaces," Duke Mathematical Journal, vol. 12, pp. 291-302, 1945. 


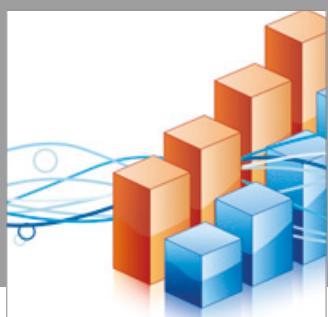

Advances in

Operations Research

vatersals

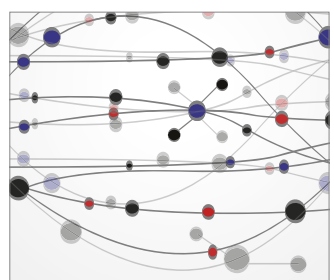

\section{The Scientific} World Journal
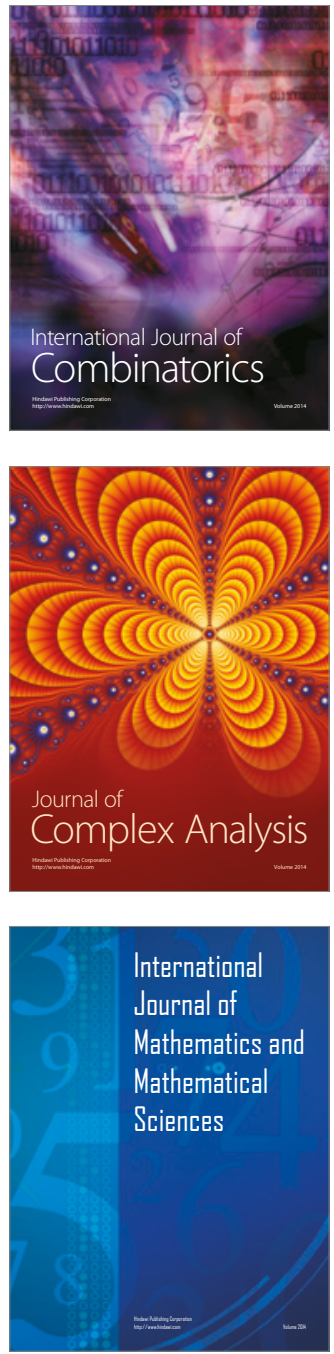
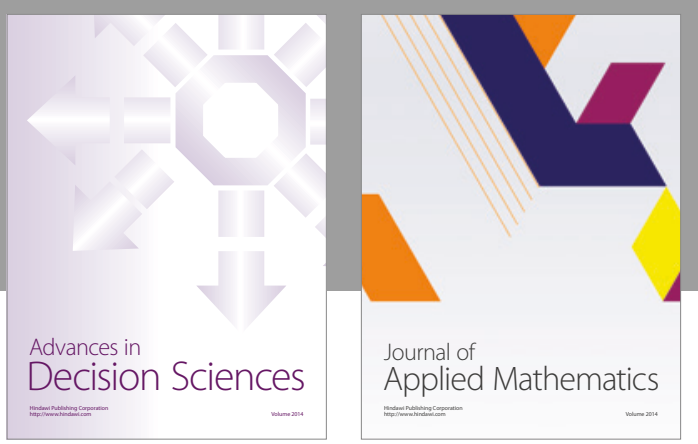

Algebra

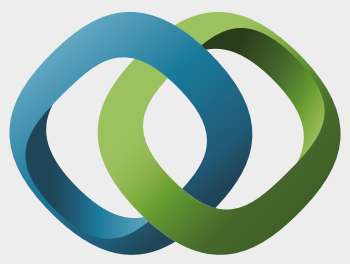

\section{Hindawi}

Submit your manuscripts at

https://www.hindawi.com
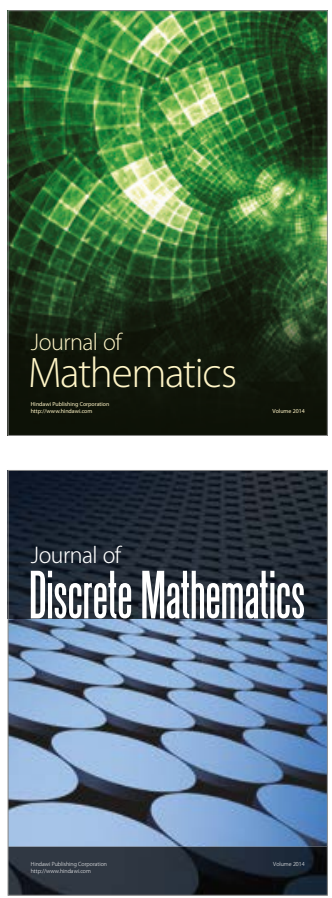

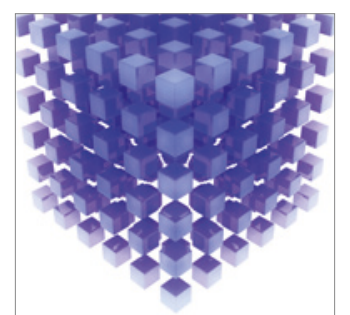

Mathematical Problems in Engineering
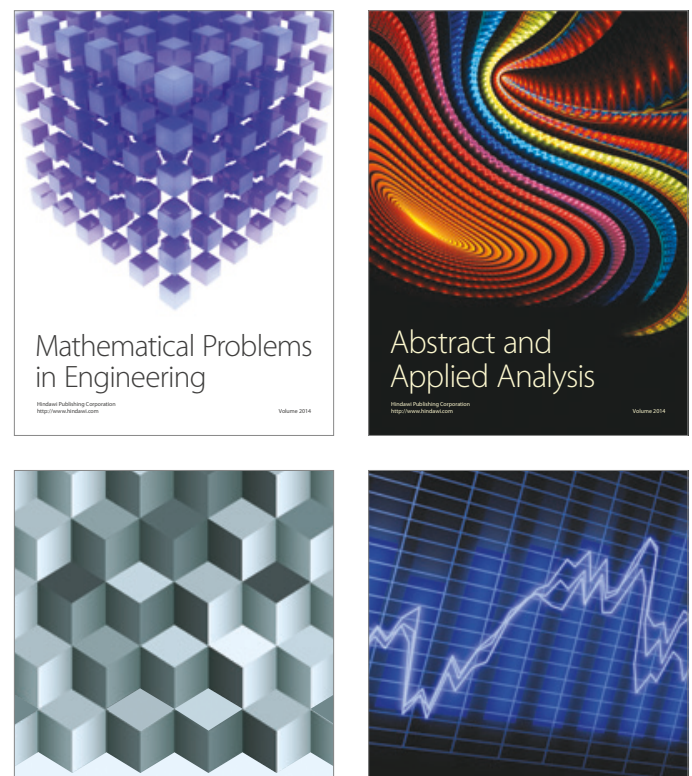

Journal of

Function Spaces

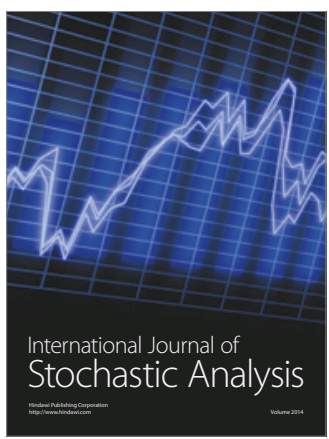

Probability and Statistics
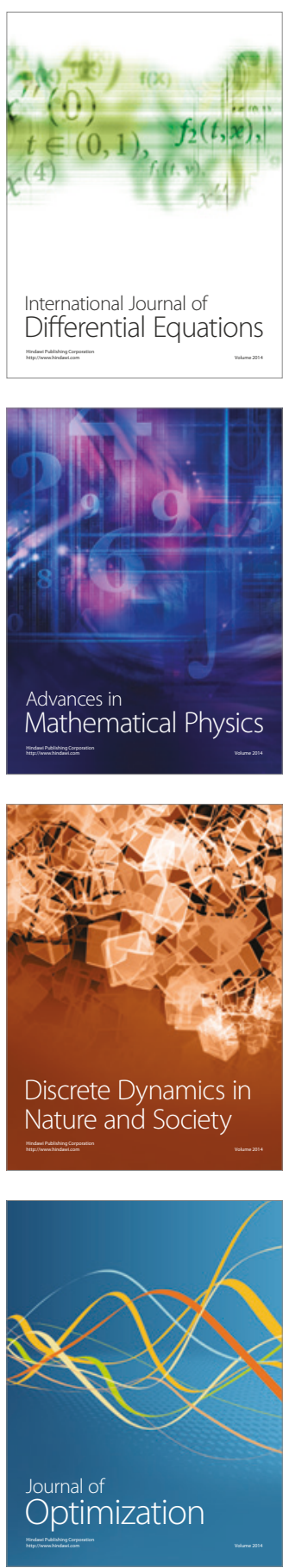